\title{
Neighbor Table Based Tree Routing in ZigBee Wireless Networks
}

\author{
Pan Tianhong ${ }^{2}$, Liu $\mathrm{Ji}^{2}$ and Mian Khuram $\mathrm{Ahsan}^{3}$ \\ School of Electrical \& Information Engineering, Jiangsu University, Zhenjiang, \\ Jiangsu 212013, China
}

\begin{abstract}
ZigBee is a kind of industrial standard of wireless personal area network with low rate and low power consumption, which is based on the physical (PHY) and medium access control (MAC) layers of IEEE 802.15.4. In ZigBee wireless networks, the tree routing $(T R)$ is widely used to data transmission because the TR does not require any routing tables and route discovery overhead. However, the TR often provides inefficient paths due to the limitation of tree topology. In this paper, an improved TR algorithm, i.e., neighbor table based tree routing (NTR), is proposed to reduce the routing hop count, shorten end to end delay and decrease packet loss rate compared with the performance of the TR. Using the proposed NTR algorithm, the source node can transmit data packets to neighbor node directly when the destination address is in the sub tree of neighbor node or neighbor node's parent. The case study demonstrates the performance of the proposed algorithm.
\end{abstract}

Keywords: ZigBee, tree routing, neighbor table, NS2 simulation

\section{Introduction}

ZigBee is a global standard of wireless personal area network targeted at providing low cost, low rate, low power consumption, flexible, and scalable wireless products [1]. It is based on IEEE 802.15.4 standard. The IEEE 802.15.4 standard defines the lower two layers: the physical (PHY) layer and the medium access control (MAC) layer. The upperlayer including the network (NWK) layer and application (APL) layer is defined by the ZigBee Alliance. ZigBee can be used as basic technology for the development of low energy and low cost Wireless Sensor Networks (WSNs). Since the characteristics of ZigBee are low-cost, low-power and low-rate, it has been used in many fields such as, home and building automation [2], health care monitoring [3], industrial control [4], environmental monitoring and surveillance [5], etc.

Routing is to discover paths from source nodes to destination nodes based on their network addresses. It is a challenging issue in Wireless Sensor Networks (WSNs) due to their inherent memory and energy constraints. In ZigBee network layer, there are two common routing protocols, i.e. Ad-hoc on demand distance vector junior (AODVjr) protocol [6] and tree routing (TR) protocol [1]. TR is a typical static routing based on distributed block addressing scheme. In ZigBee TR, each node sends data packets to the parent node or child nodes according to parent-child links without requiring any routing tables or route discovery. Therefore, TR is simple and uses resources effectively. Due to these advantages, TR is considered as a promising protocol for networks consisting of resource constrained nodes. However, TR does not provide the optimal routing path since the data packets transfer only relies on the parent-child relationship among nodes. Moreover, the nodes which near to the coordinator of the network will consume much

Received (June 18, 2016), Review Result (August 24, 2016), Accepted (December 7, 2016)

${ }^{2}$ Corresponding author: Tianhong Pan, mobile: (86)15805298357, Fax: (86)511-88790618, Email: thpan@ujs.edu.cn. 
power attributable to serious participate in data packets forwarding. Those nodes may lead to potential network segmentation and greatly affect the lifetime of the network.

In the past decades, several routing algorithms based on TR have been proposed. Harbawi et al. [7] proposed an Improved Tree Routing (ImpTR) algorithm which determined the superior path to the sink-node depending on the neighbor table. In ImpTR, the data packets are forwarded to the neighbor node if the routing hops to the destination node are less than the hops through tree routing. Mu et al. [8] proposed an adaptive routing optimization and energy-balancing algorithm by adding the neighbor information to optimize the routing paths and reducing the power cost of low-battery device. Ban et al. [9] proposed an improved ZigBee tree routing algorithm based on energy balance. The neighbor table was introduced and the residual energy of nodes was considered to achieve optimal network performance. Kim et al. [10] proposed the shortcut tree routing (STR) algorithm to achieve memory efficiency and routing efficiency. In STR, a source node (or an intermediate node) selects the optimal next hop node with the smallest remaining hop count to the destination from neighbor table. However, the STR's efficiency is dependent the number of nodes in 1-hop communication range. If the neighbor nodes become less, it may limit STR to search better shortcut. To alleviate these problems mentioned above, the neighbor table based tree routing (NTR) is proposed to reduce the number of routing hop count, achieve smaller end-to-end latency during routing, and improve the packet delivery ratio.

The rest of this paper is organized as follows. ZigBee protocol stack and TR algorithm are introduced briefly in Section 2. The technical details of NTR algorithm is presented in Section 3. The performance evaluation and conclusion are given in Section 4 and Section 5 , respectively.

\section{Related Works}

\subsection{Overview of IEEE 802.15.4/ZigBee Protocols}

The IEEE 802.15.4 standard defines the physical layer and medium access control layer, while the upper layer of the protocol stack is specified by the ZigBee Alliance. The ZigBee protocol stack is depicted in Figure 1.

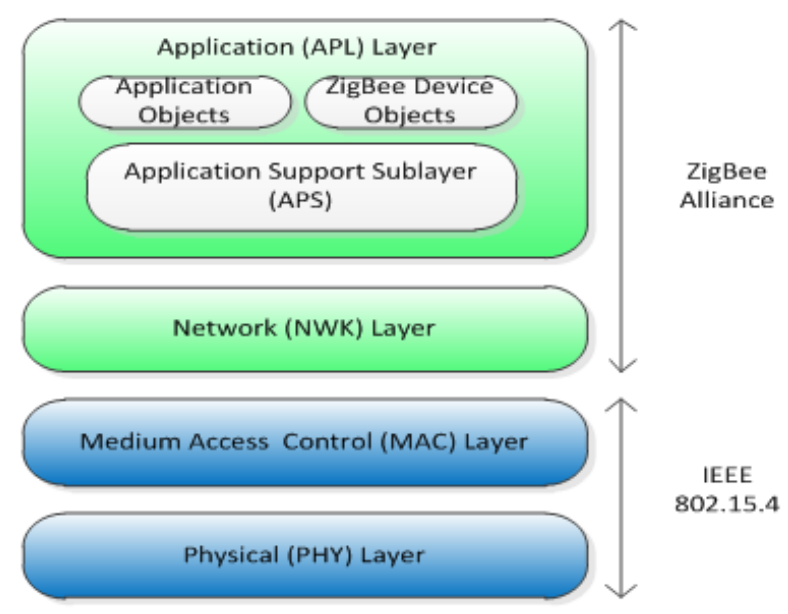

Figure 1. Zigbee Protocol Stack

It can be seen that the IEEE and ZigBee Alliance have been working closely to specify the entire protocol stack. The IEEE 802.15.4 PHY layer provides one channel for ISM $868 \mathrm{MHz}$, ten channels for ISM $915 \mathrm{MHz}$, and 16 channels for ISM $2.4 \mathrm{GHz}$. The data rate is $250 \mathrm{kbps}$ at $2.4 \mathrm{GHz}, 40 \mathrm{kbps}$ at $915 \mathrm{MHz}$ and $20 \mathrm{kbps}$ at $868 \mathrm{MHz}$. The IEEE 
802.15.4 PHY layer provides energy and link quality detection, clear channel assessment, as well as data transmission and reception. The IEEE 802.15.4 MAC layer supports two operational modes for the access to the medium: the non-beacon-enabled mode, in which the MAC is ruled by non-slotted CSMA/CA (carriers sense multiple accesses with collision avoidance); the beacon-enabled mode, in which slotted CSMA-CA mechanism shall be used. In the non-beacon-enabled network, the devices which want to transmit are allowed to send data packets whenever necessary. In a beacon-enabled network with super frame, the beacon is used to synchronize the attached devices as well as to describe the structure of the super frame and send control information to the PAN. The functions of MAC layer are beacon management, channel access, CTS (guaranteed time slot) management, association and disassociation.

The NWK layer interfaces between the MAC and the APL and is responsible for managing network formation and routing. The IEEE 802.15.4 standard defines two types of network nodes: full function device (FFD) and reduced function device (RFD). While ZigBee defines three different device types: Personal Area Network (PAN) coordinator, router, and end device. The PAN coordinator must be a FFD that is responsible for initializing, maintaining, and controlling the network. A ZigBee router is a FFD with the capability of routing and supporting associations. A ZigBee end device is a RFD or FFD which must be associated with a FFD. ZigBee networks supports star, tree and mesh topologies. In a star network, the network is controlled by one device called the ZigBee coordinator and other devices, known as end devices, directly communicate with the ZigBee coordinator. For tree and mesh networks, devices can communication with each other in a multi-hop fashion and the network may be extended through the use of routers. The tree network can operate on beacon-enabled (with synchronization) and non-beaconenabled (without synchronization) modes. Figure 2 shows examples of these topologies.

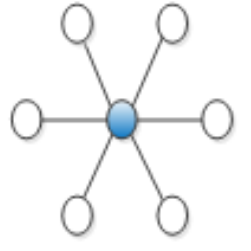

Star

PAN coardinatoriffD

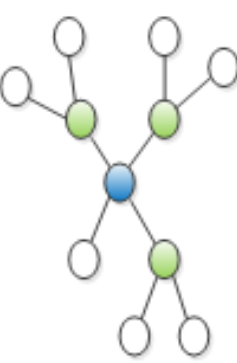

Tree

Router[FF]

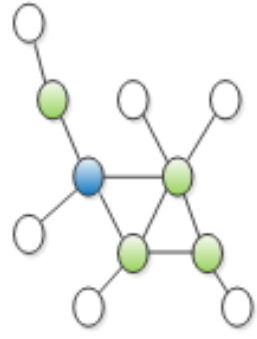

Mesh

End device(RFD)

Figure 2. Network Topologies in ZigBee

The application (APL) layer is the highest protocol layer in the ZigBee protocol stack and consists of the APS sub layer, the ZDO and the manufacturer-defined application objects. Manufactures develop the application objects to customize a device for various applications. Application objects control and manage the protocol layers in a ZigBee device. There can be up to 240 applications objects in a single device.

\subsection{Distributed Address Assignment Mechanism}

As mentioned before, there are two algorithms in ZigBee NWK layer, i.e. AODVjr routing and Tree routing. AODVjr is one of the table-driven routing protocols which provide efficient paths for the arbitrary source and destination pair. However, the route discovery overhead and the memory consumption proportionally increase with the number of route discovery. On the other hand, in TR, each node only relays data packets 
to the parent node or one of the child nodes by comparing its address with the destination address.

There are two address modes in ZigBee networks: 64-bit long address and 16-bit short address. The 64-bit long address, also called IEEE or MAC address, is assigned by the manufacturer. And the 16-bit short address, which is also called network address, is unique within a particular network and will be assigned by a parent node to its children nodes. In ZigBee specification, the distributed address assignment mechanism has been defined for assigning the short address, which will be used in routing mechanism. Each potential parent node is provided with a limited sub-block address space which is used to assign network address to its child nodes. Given values for the maximum number of children $(\mathrm{Cm})$ a parent may have, the maximum number of routers $(\mathrm{Rm})$ a parent may have as children, and the maximum depth of the network ( $\mathrm{Lm}$ ), the range of the address space of parent node at depth $\mathrm{d}$ is formulated as

$$
\text { Cskip }(d)= \begin{cases}1+C m \times(L m-d-1), & \text { if } R m=1 \\ \frac{1+C m-R m-C m \times R m^{L m-d-1}}{1-R m}, & \text { otherwise }\end{cases}
$$

The network address $A_{d+1, r n}$ and $A_{d+1, e l}$ are allocated to the $n^{\text {th }}$ router and $l^{\text {th }}$ end device which at depth $d+1$, respectively, according to the following equations

$$
\begin{gathered}
A_{d+1, r n}=A_{\text {parent }}+\operatorname{Cskip}(d) \times(n-1)+1 \\
A_{d+1, e l}=A_{\text {parent }}+\operatorname{Cskip}(d) \times R m+l
\end{gathered}
$$

where $\mathrm{A}_{\text {parent }}$ represents the address of the parent at tree level $\mathrm{d}$ and $1 \leq n \leq R m$, $1 \leq l \leq C m-R m$.

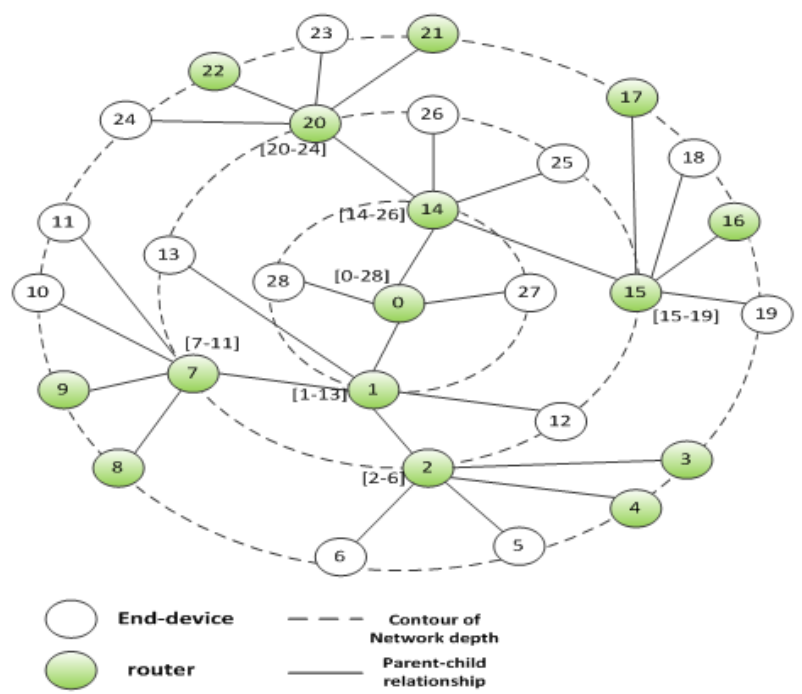

Figure 3. Network Address Allocation

Figure 3 gives an example of the network address allocation with $R m=2, C m=4$, and $L m=3$. All addresses have been assigned to routers and end devices. The address appears inside the circle representing each node, while the assigned address ranges are displayed in brackets close to each router.

\subsection{Tree Routing Algorithm}

In tree routing algorithm, when a node receives a packet, the routing of the packet is decided by several certain rules. Here, it is assumed that the address of current router is A 
at a depth of $d$ and the packet will be sent to the node whose address is $\mathrm{D}$, then the tree routing process can be summarized as follows:

Step 1: Judge the destination node of data packets is the node self. If yes, data is delivered to the upper layer directly; or else, go to Step 2;

Step 2: Check the destination node is one of its descendant nodes by using Eq. (4).

$$
A<D<A+C \operatorname{cskip}(d-1)
$$

If yes, the next hop node $A_{\text {next }}$ is calculated

$$
A_{\text {next }}= \begin{cases}D, & \text { if } D>A+R m \times \operatorname{Cskip}(d) \\ A+1+\left\lfloor\frac{D-(A+1)}{\operatorname{Cskip}(d)}\right\rfloor \times C \operatorname{Cskip}(d), & \text { otherwise }\end{cases}
$$

Or else, the packet is relayed to its parent.

For instance, Figure 3 is an example of tree topology where $R m=2, C m=4$ and $L m=3$. Assume that the source is node 8 and the destination is node 2. According to TR, the source judges the destination whether is its descendant by Eq.(4). Since the destination is not the descendant of the source, the next hop is the source's parent node (node 7). Node 7 needs to implement step 1 and step 2 for determining the next hop. As a result, node 1 is selected as the next hop. Node 1 implements the same procedure as node 7 . Finally, node 1 discovers that the destination (node 2) is its child node, so the data packets are sent directly to node 2 .

\subsection{Problem Description}

TR algorithm is relatively simple because it only takes the relationship between parent and child nodes into consideration and doesn't include the routing table. However, the detour path problem cannot be avoided. An example is shown in Figure 4. Supposing the source node (S) sending data to destination node (D), in accordance with the original tree routing algorithm, the data packets should be sent to the root node following the parent node $(S \rightarrow 1 \rightarrow 0)$, and goes back to the destination node $(0 \rightarrow 2 \rightarrow \mathrm{D})$. According to this method, the data packets have to experience four hops from the source node to the destination node. However, if neighbor nodes are considered, the source node send the data packets directly to the destination node, which needs only one hop (solid arrow in Figure 4).

Moreover, in ZigBee TR, most of the traffic will go through the nodes which are close to the coordinator and cause these nodes excessive energy consumption. In order to solve problems mentioned above, neighbor nodes should be the candidate nodes as next hop node.

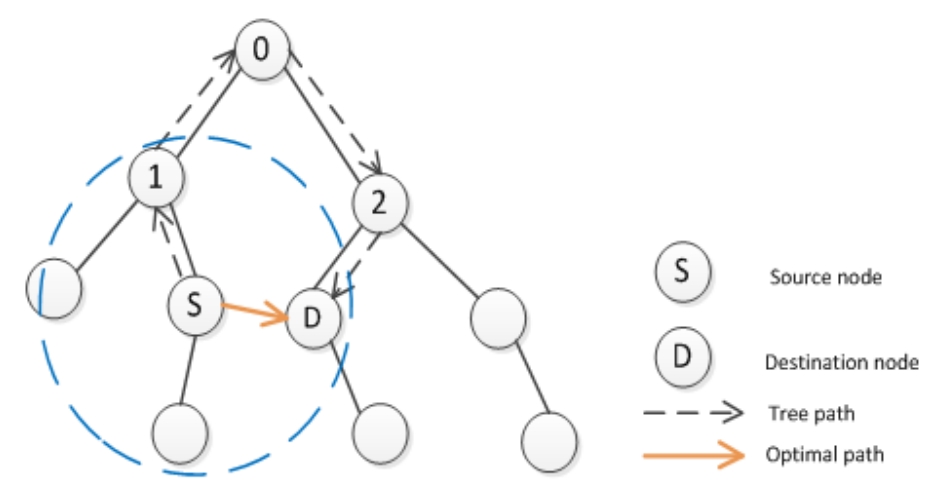

Figure 4.Tree Routing 


\section{Neighbor Table Based Tree Routing Algorithm}

To reduce possible detour path and the energy consumption of the nearest nodes to coordinator, a neighbor table based tree routing (NTR) is proposed. The NTR algorithm takes the idea from the ZigBee TR, but chooses one of neighbor nodes (excluding parent or children) as the next hop node when the destination node is in the sub-tree of neighbor node or neighbor node's parent. An example of routing determination by the NTR algorithm is shown in Figure 5. The NTR compares whether the address of the destination node (D) is contained in the address space of neighbor node or neighbor node's parent, and selects the N1 as the next hop node to forward the data packets to the destination node.

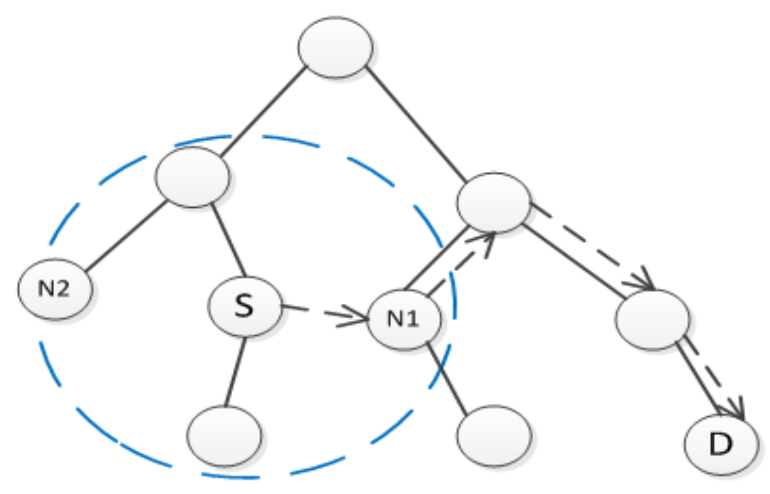

Figure 5. Neighbor Table-based Tree Routing

Compared to the TR, the link of the neighbor nodes provides the efficient path. In TR, the number of hops [12] between source node (S) and destination node (D) is calculated by

$$
N H(S, D)=\operatorname{Depth}(S)+\operatorname{Depth}(D)-2 \operatorname{Depth}(F C A(S, D))
$$

Where $N H(S, D)$ is the number of hops between source node and destination node, $\operatorname{Depth}(\mathrm{X})$ is the depth of node $\mathrm{X}$, and $F C A(S, D)$ is the first common ancestor node of nodes $\mathrm{S}$ and D. Because the NTR algorithm follows fundamental TR, the hop count between source node and destination node can be calculated by Eq. (6). We define $N H_{n}(S, D)$ and $N H_{n p}(S, D)$ as the routing cost of NTR when the destination node is in the address space of neighbor node and neighbor node's parent, respectively. That is

$$
\begin{gathered}
N H_{n}(S, D)=\operatorname{Depth}(D)-\operatorname{Depth}(N)+1 \\
N H_{n p}(S, D)=\operatorname{Depth}(D)-\operatorname{Depth}\left(N_{n p}\right)+2
\end{gathered}
$$

Where $N$ and $N_{n p}$ are the address of the neighbor node and neighbor node's parent, respectively. In Eq. (8), it is assumed that the neighbor node is not sibling node and the packet goes along the ZigBee tree routing. In other words, the proposed algorithm calculates the routing cost in the same way the TR does. Since the next hop node is neighbor node, the numbers of hops between source node and neighbor node or neighbor node's parent according to original tree routing are at least 2-hops .The ZigBee tree routing cost between $\mathrm{S}$ and $\mathrm{D}$ is also expressed as follows:

$$
\left\{\begin{array}{l}
N H_{n}^{\prime}(S, D) \geq \operatorname{Depth}(D)-\operatorname{Depth}(N)+2, \text { if } N \leq D<N+C \operatorname{skip}\left(d_{n}-1\right) \\
N H_{n p}^{\prime}(S, D) \geq \operatorname{Depth}(D)-\operatorname{Depth}\left(N_{n p}\right)+2 \text {, if } N_{p} \leq D<N_{p}+C \operatorname{sip}\left(d_{n p}-1\right)
\end{array}\right.
$$


Where $d_{n}$ and $d_{n p}$ represent the depth of neighbor node and neighbor node's parent, respectively. It is obvious that the routing path by the NTR is more efficient than the ZigBee tree routing algorithm.

The procedure of the NTR algorithm is as follows:

Step 1: Judge the destination node is it's descendent or not (using Eq. (4)). If yes, data packets will be sent to its child node; or else, jump to Step2.

Step 2: Check the destination node is the neighbor node using node's neighbor table or not. If yes, the data packets will be delivered directly to the destination node; or else, jump to Step 3.

Step 3: Check the address of destination node is in the address space of neighbor node or not (using Eq. (10))

$$
\begin{cases}N<D, & \text { if } d_{n}=0 \\ N<D<N+C \operatorname{skip}\left(d_{n}-1\right), & \text { if } d_{n} \geq 1\end{cases}
$$

If yes, the neighbor node is selected as the candidate node for next hop. Furthermore, if there are several neighbor nodes satisfy Eq. (10), the neighbor node with maximum tree depth is selected as the next hop node. Or else, jump to Step 4.

Step 4: If $d_{n}>0$, check the address of the destination node is in the sub tree of the neighbor node's parent or not (using Eq. (11))

$$
\begin{cases}N_{n p}<D, & \text { if } d_{n}=1 \\ N_{n p} \leq D<N_{n p}+C \operatorname{skip}\left(d_{n p}-1\right), & \text { if } d_{n}>1\end{cases}
$$

Where $N_{n p}$ should be computed, if $d_{n}=1, N_{n p}=0$; otherwise, following equations will be executed

$$
\left\{\begin{array}{l}
A_{i+1}=A_{i}+1+\left\lfloor\frac{N-\left(A_{i}+1\right)}{\operatorname{Cskip}(i)}\right\rfloor \times \operatorname{Cskip}(i), \\
i=i+1,
\end{array}\right.
$$

Where $A_{i}$ represent the address of neighbor node's parent at depth $i$ and $A_{0}$ is equal to 0 . Repeat executing Eq. (12) until $i=d_{n}-2$. Define one variable: $A_{s p}$ (the address of current node's parent). If conditions $A_{s p} \neq N_{n p}$ and Eq. (11) is met, corresponding neighbor node will be the candidate node for the next hop. Choosing correspond neighbor node whose parent has the maximum tree depth from candidate nodes as the next hop node. If there is no candidate node, jump to Step 5.

Step 5: Data packets will be directly transmitted to its parent.

Repeat Steps 1-5 for each source node or intermediate node until data packets have been sent to destination node.

\section{Performance Evaluation}

The network simulator NS-2 is used for comparing NTR with TR. The simulation parameters and their value are summarized in Table 1. Three metrics of routing performance: average hop counts, average end-to-end delay and average packet loss rate, are taken for comparison. In the simulation, the coordinator is located at the center of the topology of $100 \mathrm{~m} \times 100 \mathrm{~m}$, while other nodes are randomly deployed. Both the source and the destination nodes are chosen randomly in each network topology $(\mathrm{CBR}$ traffic $=10)$. Because both the source and the destination nodes are different in each test, we measure those metrics after repeating each test over ten times. 
Table 1. Parameters Used in Simulation

\begin{tabular}{|c|c|}
\hline Network size & $100 \mathrm{~m} \times 100 \mathrm{~m}$ \\
\hline PHY/MAC protocol & IEEE 802.15 .4 \\
\hline Propagation model & Two-ray ground \\
\hline Transmission range & $20 \mathrm{~m}$ \\
\hline Interface queue/size & Priority queue/80 \\
\hline Network protocol & NTR/TR \\
\hline Cm, Rm, Lm & $4,4,5$ \\
\hline Simulation time & $400 \mathrm{sec}$ \\
\hline Packet size & 80 byte \\
\hline Packet type & CBR \\
\hline
\end{tabular}

Figure 6 shows the average hop count from source to destination with increasing number of nodes. The performance evaluation of ZigBee tree routing and our NTR is performed in the same network topology. As the number of nodes grows, the average hop count does not increase linearly because the source and destination nodes are randomly selected and the network topology is different in each test. However, the average hop count of the NTR is much smaller than that of ZigBee tree routing. As Figure 6 shows, the average hop count per pair of source and destination nodes of the NTR is 1-2 hops less than that of ZigBee tree routing. This is because the source node has to forward over many hops to relay the data to the destination if the routing follows the regular ZigBee tree routing. In NTR algorithm, if the destination address is in the sub tree of neighbor node or neighbor node's parent, the source (or intermediate) node selects the neighbor node instead of its parent as the next hop node. Thus, the average hop count of NTR algorithm is smaller than that of TR algorithms.

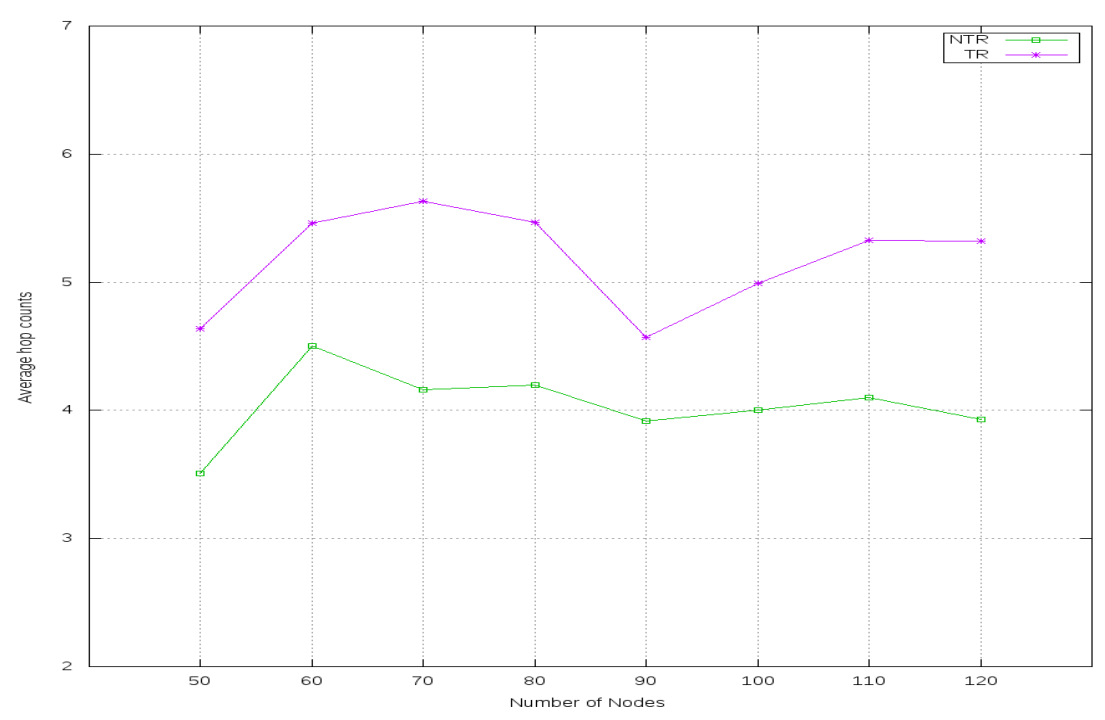

Figure 6. Average Hop Counts

Figure 7 shows that the proposed NTR's latency is lower than that of the ZigBee tree routing. Moreover, the average end-to-end delay per pair of source and destination nodes in the ZigBee tree routing increases as the number of nodes in the network increase (excluding number of nodes equal ninety); the NTR algorithm, however, is less affected by the number of nodes in the network. This result indicates that a denser network causes longer latency, since more nodes are deployed. When the number of nodes increases, the 
performance improvement is more significant. In TR algorithm, the node which is near to the coordinator of the network relay more packets, it may lead to more congestion and collision of packets because nodes compete for channel access to transmit the data packets. The proposed NTR algorithm attempts to reduce the hop count by adding neighbor information during the transmission of the data packets. In addition, the neighbor node selected as the next hop node shares the traffic load of parent nodes in high tree levels. As a result, NTR has smaller end-to-end delay compared with TR.

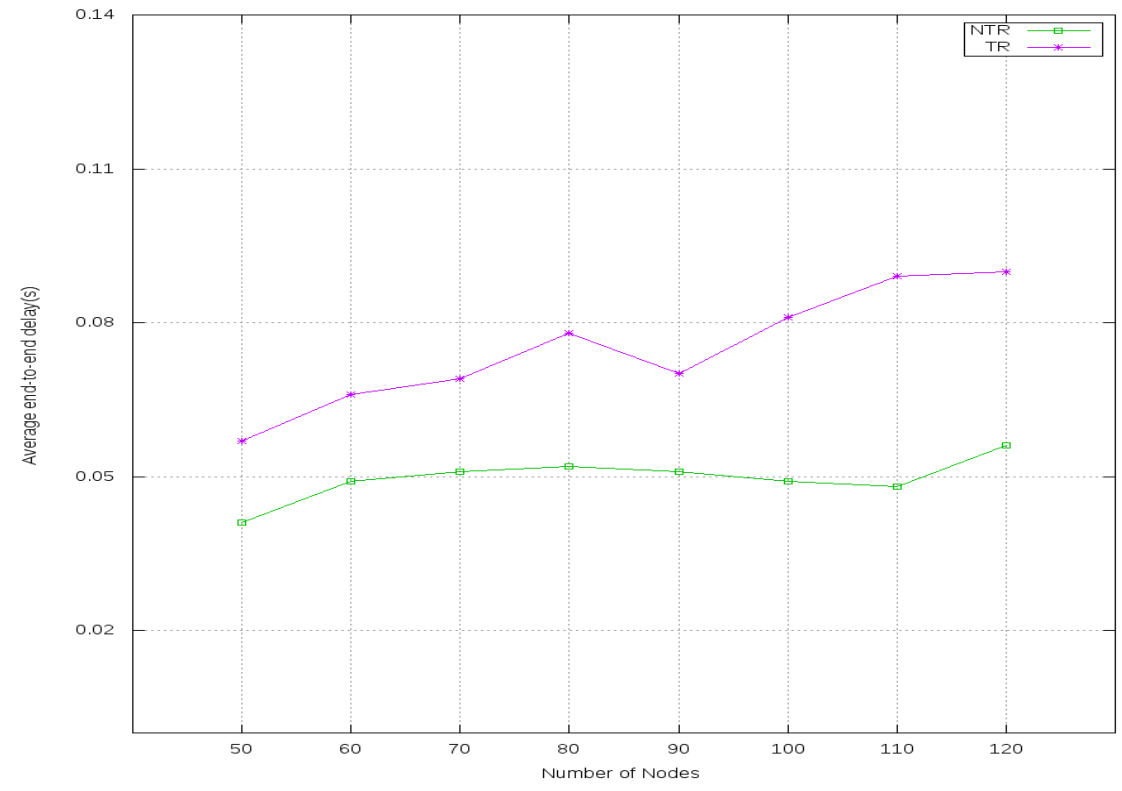

Figure 7. Average End-to-end Delay

The average packet loss rate tends to increase as the number of nodes in the network grows, and it is natural in that collision and contention of packets increase for the increasing network density. As shown in Figure 8, the NTR algorithm provides reliable communication than TR. It is because the NTR algorithm can choose a neighbor node as the next hop to cut across the tree topology, relieve congestion and decrease packet loss rate. Thus, the NTR algorithm can improve the ZigBee's performance in terms of packet loss rate compared to the TR. 


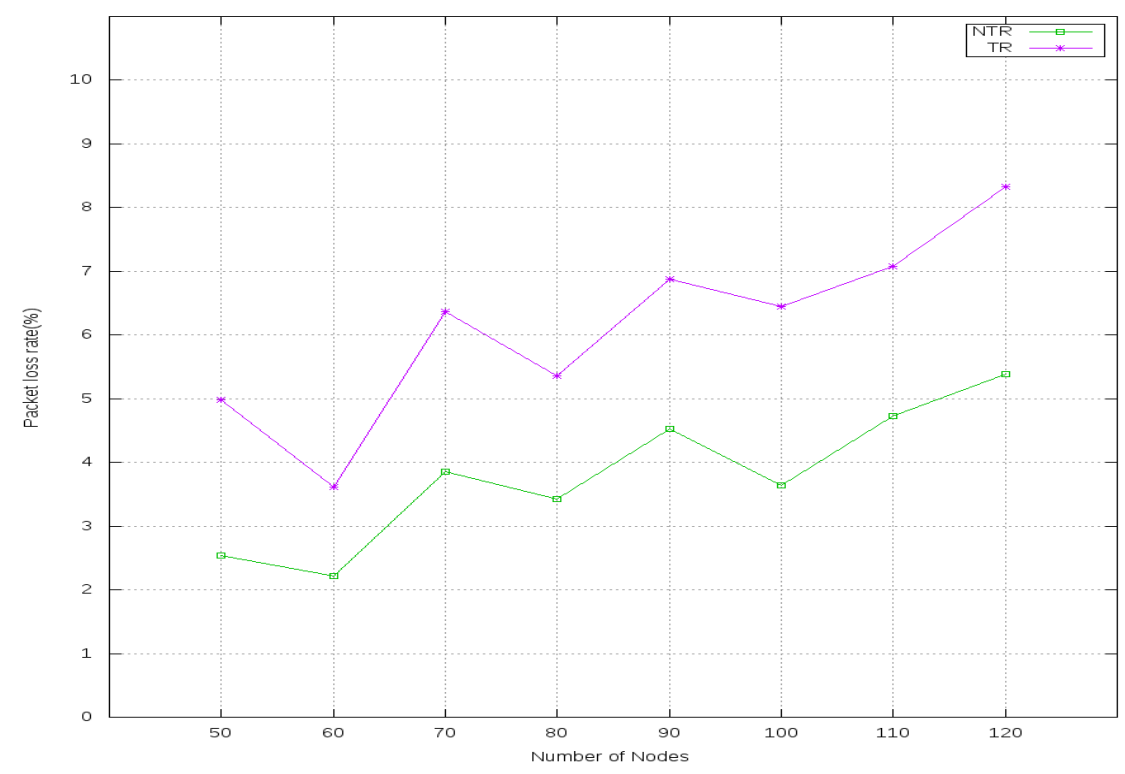

Figure 8. Average Packet Loss Rate

\section{Conclusion}

In this paper, the NTR algorithm is proposed to overcome the detour path problem and traffic concentration problem of the ZigBee tree routing. In NTR, each node can find the optimal next hop node by using their neighbor table, originally defined in the ZigBee standard. The mathematical analyses prove that the one hop neighbor information in NTR can provide an efficient routing path. The NTR improves the routing efficiency of original tree routing and keeps the advantage of the tree routing without any routing discovery. The simulation results show that the NTR can effectively reduce the redundant routing hops of TR, achieve smaller end-to-end delay than TR during routing, and improve the reliability of the network data transmission. Therefore, it is expected that NTR to be utilized in many ZigBee applications with limited resources.

\section{Acknowledgement}

The authors would like to thank the financial support provide by the National Natural Science Foundation under Grant 61273142, Foundation for Six Talents by Jiangsu Province (2012-DZXX-045), Priority Academic Program Development of Jiangsu Higher Education Institutions (PAPD), and Technology research project of Ministry of public security of China (grant number 2015JSYJC029).

\section{Conflict of Interest}

The authors indicated no potential conflicts of interest.

\section{References}

[1] ZigBee Alliance, "ZigBee Specification", (2009).

[2] J. Byun, B. Jeon, J. Noh, Y. Kim and S. Park, "An intelligent self-adjusting sensor for smart home services based on ZigBee communications", IEEE Transactions on Consumer Electronics, vol. 58, no. 3, (2012), pp. 794-802.

[3] N. J. LaSorte, S. A. Rajab and H. H. Refai, "Developing a reproducible non-line-of-sight experimental setup for testing wireless medical device coexistence utilizing ZigBee", IEEE Transactions on Biomedical Engineering, vol. 59, no. 11, (2012), pp. 3221-3229. 
[4] S. Yoo, P. K. Chong and D. Kim, "Guaranteeing real-time services for industrial wireless sensor networks with IEEE 802.15. 4", IEEE Transactions on Industrial Electronics, vol. 57, no. 11, (2010), pp. 3868-3876.

[5] W. T. Sung and M. H. Tsai, "Multi-Sensor wireless signal aggregation for environmental monitoring system via multi-bit data fusion", Applied Mathematics \& Information Sciences, vol. 5, no. 3, (2011), pp. 589-603.

[6] I. D. Chakeres and L. K. Berndt, "Aodvjr, aodv simplified”. ACM SIGMOBILE Mobile Computing and Communications Review, vol. 6, no. 3, (2002), pp. 100-101.

[7] M. K. A. Al-Harbawi, M. F. A. Rasid and N. K. Noordin, "Utilizing Neighbours-Table to Improve Tree Routing Protocol in ZigBee Network", Wireless Personal Communications, vol. 65, no. 2, (2012), pp. 469-488.

[8] J. Mu, W. Wang, B. Zhang and W. Song, "An adaptive routing optimization and energy-balancing algorithm in ZigBee hierarchical networks", EURASIP Journal on Wireless Communications and Networking, vol. 43, no. 1, (2014), pp. 1-11.

[9] Y. L. Ban, Q. L. Chai and W. Chen, "ZigBee tree routing algorithm based on energy balance: ZigBee tree routing algorithm based on energy balance", Journal of Computer Applications, vol. 28, no. 28, (2008), pp. 2791-2794.

[10] T. Kim, D. Kim and N. Park, "Neighbor table based shortcut tree routing in ZigBee wireless networks", IEEE Transactions on Parallel and Distributed Systems, vol. 25, no. 3, (2014), pp. 706-716.

[11] J. Y. Ha, H. S. Park, S. Choi and W. H. Kwon, "EHRP: Enhanced hierarchical routing protocol for zigbee mesh networks ee", Communications Letters, IEEE, vol. 11, no. 12, (2007), pp. 1028-1030.

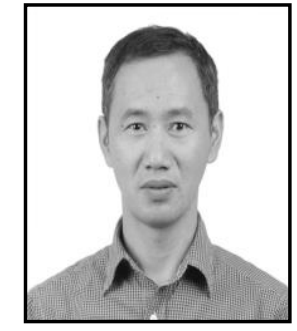

Pan Tianhong, received his B.S. degree from Anhui Agriculture University and M.S. degree from Gansu University of Technology in 1997 and 2000 respectively. And he received his Ph.D. degree in control theory and control engineering from Shanghai Jiao Tong University in 2007. Now he has been a professor in School of Electrical and Information Engineering, Jiangsu University, Zhenjiang, China. His current research interests include multiple model approach and its application, predictive control and run-to-run control theory and practice, system identification, etc.

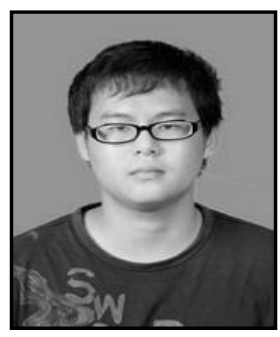

Liu Ji, he received the B.S. degree from Jiangsu University in 2014. He is currently pursuing M.S. degree with the School of Electrical and Information Engineering, Jiangsu University, Zhenjiang, China. His current research interests include wireless communication and sensor networks.

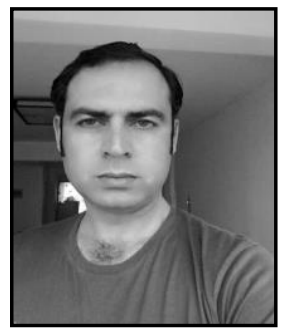

Mian Khuram Ahsan, is currently pursuing Ph.D. degree with the School of Electrical and Information Engineering, Jiangsu University, Zhenjiang, China. His current research interests include micro-grid system, multi-agent algorithm, \& wireless communication and sensor networks. 
International Journal of Control and Automation Vol.10, No.9 (2017) 